\title{
Accessing high momentum nucleons in lattice QCD
}

\author{
Jia-jun $\mathbf{W u}^{*}$ \\ Special Research Centre for the Subatomic Structure of Matter (CSSM), \\ Department of Physics, University of Adelaide, Australia \\ E-mail: jiajun.wu@adelaide.edu.au

\section{Waseem Kamleh} \\ Special Research Centre for the Subatomic Structure of Matter (CSSM), \\ Department of Physics, University of Adelaide, Australia \\ E-mail: waseem.kamleh@adelaide.edu.au

\section{B. Leinweber} \\ Special Research Centre for the Subatomic Structure of Matter (CSSM), \\ Department of Physics, University of Adelaide, Australia \\ E-mail: derek.leinweber@adelaide.edu.au
}

\section{R. D. Young}

Special Research Centre for the Subatomic Structure of Matter (CSSM),

Department of Physics, University of Adelaide, Australia

E-mail: ross.young@adelaide.edu.au

\section{J. M. Zanotti}

Special Research Centre for the Subatomic Structure of Matter (CSSM),

Department of Physics, University of Adelaide, Australia

E-mail: james.zanotti@adelaide.edu.au

\begin{abstract}
A stochastic source using a dilute grid of source points and incorporating momentum-based smearing is used to generate the correlation function of the proton at rest and in a boosted frame. The dependence of the error terms on the number of source points and source separation is studied. Calculations on a $24^{3} \times 48$ lattice demonstrate that this novel technique yields reduced statistical errors for the proton correlation function in boosted frames, and can be used to successfully extract precise values of the effective energy at high momentum.
\end{abstract}

The 26th International Nuclear Physics Conference

11-16 September, 2016

Adelaide, Australia

${ }^{*}$ Speaker. 


\section{Introduction}

Lattice QCD has achieved great success in understanding hadronic structure via a first principles approach to calculating the properties of QCD, in particular the hadron spectrum $[1,2]$. However, when studying baryonic properties the exponential increase of statistical noise with Euclidean time continues to prove a challenge, especially for boosted systems at high momentum values. The study of hadrons carrying momentum is an important topic in various applications, including hadronic form factors.

Due to the increasing statistical errors, the use of standard lattice techniques to study hadrons at high momenta has proven difficult. However, recently there has been significant progress in this area. The use of a parity-expanded variational analysis [3] eliminates cross-parity contaminations at finite momenta, enabling clean extraction of excited state energies. At very large values of momenta extracting even the ground state energy can prove challenging. Here, the use of a novel quark smearing utilising momentum phases [4] has proven advantageous in maintaining small statistical errors.

In this study, we apply momentum-mearing to a stochastic source to enhance the signal of the proton at high momentum. Stochastic source techiques are commonly used for all-to-all propagators $[5,6,7,8,11]$, and for mesons, through the additional volume averaging provided by the "one-end trick" $[9,10]$, have been shown to provide an improved signal when compared with a standard point-to-all propagator. Here, we use the baryonic equivalent of the one-end trick to introduce a dilute stochastic source constructed with $\mathbb{Z}_{3}$ noise to extract the effective energy of a proton at rest and at finite momentum. Furthermore, we will emphasize how to choose the dilute source locations to optimise the correlation function signal.

\section{Framework}

\subsection{Proton correlation function}

The standard lattice operator for the proton is

$$
\phi(\vec{x}, t) \equiv \varepsilon^{a b c}\left(u^{a T}(\vec{x}, t) C \gamma_{5} d^{b}(\vec{x}, t)\right) u^{c}(\vec{x}, t),
$$

yielding the corresponding two-point correlation function,

$$
\begin{aligned}
G(\vec{x}, t, \vec{p}, \Gamma) & \equiv \operatorname{Tr} \sum_{\vec{y}} \Gamma e^{i \vec{q} \cdot(\vec{y}-\vec{x})}\langle T(\phi(\vec{y}, t), \bar{\phi}(\vec{x}, 0))\rangle \\
& =\sum_{\vec{y}} e^{i \vec{p} \cdot(\vec{y}-\vec{x})} \Gamma^{\gamma \gamma^{\prime}} h_{\gamma^{\prime} \gamma}\left(S_{u}(\vec{y}, t ; \vec{x}, 0), S_{d}(\vec{y}, t ; \vec{x}, 0), S_{u}(\vec{y}, t ; \vec{x}, 0)\right)
\end{aligned}
$$

The quark propagators $S_{f}$ for each flavor $u, d$ combine according to the Wick contractions,

$$
h_{\gamma \gamma^{\prime}}\left(S_{1}, S_{2}, S_{3}\right)=\varepsilon^{a b c} \varepsilon^{e f g}\left\{\operatorname{Tr}\left[S_{1}^{a e} \gamma_{5} C S_{2}^{b f T} C \gamma_{5}\right] S_{3 \gamma \gamma^{\prime}}^{c g}+\left[S_{1}^{a e} \gamma_{5} C S_{2}^{b f T} C \gamma_{5} S_{3}^{c g}\right]_{\gamma \gamma^{\prime}}\right\},
$$

where $S_{u}(\vec{y}, t ; \vec{x}, 0)=\langle T(u(\vec{y}, t), \vec{u}(\vec{x}, 0))\rangle$, Roman indices $a-g$ are for color and Greek indices $\gamma, \gamma^{\prime}$ are for spin. Here we assume iso-spin symmetry $S_{u} \equiv S_{d}$ for the nucleon. The parity projection matrix $\Gamma$ is chosen as $\left(I+\gamma_{4}\right) / 2$. 


\subsection{Dilute $\mathbb{Z}_{3}$ noise source}

Here, the one-end trick for baryons is implemented by introducing a dilute $\mathbb{Z}_{3}$ noise vector,

$$
\eta\left(\vec{x}_{i}\right) \equiv e^{i 2 R(i) \pi / 3},
$$

where $\vec{x}_{i}$ is the $i$-th source location, and $R(i)$ is a random number in the set $\{0,1,2\}$. The $\mathbb{Z}_{3}$ noise vector satisfies $\left\langle\eta\left(\vec{x}_{i}\right) \eta\left(\vec{x}_{j}\right) \eta\left(\vec{x}_{k}\right)\right\rangle=\delta_{i j} \delta_{j k}$. Hence, we can define a solution vector $\chi(\vec{y}, t)$ such that

$$
\chi(\vec{y}, t)=\sum_{i=1}^{N} S\left(\vec{y}, t ; \vec{x}_{i}, 0\right) \eta\left(\vec{x}_{i}\right)
$$

where the sum enumerates the $N$ different source locations. The source is dilute, so $N$ will be much less than the lattice volume. The spatial noise vector $\eta\left(\vec{x}_{i}\right)$ is replicated for each spin and color source index such that the quark propagator $S\left(\vec{y}, t ; \vec{x}_{i}, 0\right)$ is given by

$$
S_{\alpha \beta}^{a b}\left(\vec{y}, t ; \vec{x}_{i}, 0\right)=\left\langle\chi_{\alpha \beta}^{a b}(\vec{y}, t) \eta^{*}\left(\vec{x}_{i}\right)\right\rangle .
$$

\subsection{Momentum smearing and phase in the source}

We apply iterative momentum smearing [4] to our lattice operators to improve the signal at high momentum. Furthermore, as the stochastic propagator $\chi$ includes various source locations, it is necessary to include the appropriate the Fourier phase at nonzero momentum at each source point. It is convenient to introduce one momentum variable for each quark propagator, such that the new propagator $\chi(\vec{y}, t, \vec{q})$ can be defined as

$$
\chi(\vec{y}, t, \vec{q})=\sum_{i=1}^{N} \eta\left(\vec{x}_{i}\right) e^{-i \vec{q} \cdot \vec{x}_{i}} S_{\vec{q}}\left(\vec{y}, t ; \vec{x}_{i}, 0\right),
$$

where $S_{\vec{q}}$ is the propagator defined in Ref. [4] with momentum smearing phase $\vec{q}$ applied at the source and sink. The exponential factor $e^{-i \vec{q} \cdot \vec{x}_{i}}$ is the Fourier phase appropriate to each of the $N$ different source locations in the correlation function, and is independent of the smearing algorithm.

\subsection{Proton correlation function with momentum-based noise source}

The correlation function of the proton is calculated using the stochastic propagator $\chi(\vec{y}, t, \vec{p})$, instead of $S(\vec{y}, t ; \vec{x}, 0)$. Correspondingly, we only need to keep track of the Fourier phase at the sink, as the source phase has already been absorbed into the stochastic propagators. The new proton two-point function is given by

$$
\begin{aligned}
& G_{N}\left(t, \vec{q}_{1}+\vec{q}_{2}+\vec{q}_{3}, \Gamma\right) \\
\equiv & \sum_{\vec{y}} e^{i\left(\vec{q}_{1}+\vec{q}_{2}+\vec{q}_{3}\right) \cdot \vec{y}} \Gamma^{\gamma \gamma^{\prime}} h_{\gamma \gamma^{\prime}}\left(\chi\left(\vec{y}, t, \vec{q}_{1}\right), \chi\left(\vec{y}, t, \vec{q}_{2}\right), \chi\left(\vec{y}, t, \vec{q}_{3}\right)\right) \\
= & \sum_{\vec{y}} \sum_{i=1}^{N} e^{i\left(\vec{q}_{1}+\vec{q}_{2}+\vec{q}_{3}\right) \cdot\left(\vec{y}-\vec{x}_{i}\right)} \Gamma^{\gamma \gamma^{\prime}} h_{\gamma \gamma^{\prime}}\left(S_{\vec{q}_{1}}\left(\vec{y}, t ; \vec{x}_{i}, 0\right), S_{\vec{q}_{2}}\left(\vec{y}, t ; \vec{x}_{i}, 0\right), S_{\vec{q}_{3}}\left(\vec{y}, t ; \vec{x}_{i}, 0\right)\right) \\
& +\sum_{\vec{y}} e^{i\left(\vec{q}_{1}+\vec{q}_{2}+\vec{q}_{3}\right) \cdot \vec{y}} \sum_{i, j, k=1}^{N}\left(1-\delta_{i j} \delta_{i k}\right) \eta\left(\vec{x}_{i}\right) \eta\left(\vec{x}_{j}\right) \eta\left(\vec{x}_{k}\right) \\
& \times e^{i\left(\vec{q}_{1} \cdot \vec{x}_{i}+\vec{q}_{2} \cdot \vec{x}_{j}+\vec{q}_{3} \cdot \vec{x}_{k}\right)} \Gamma^{\gamma \gamma^{\prime}} h_{\gamma \gamma^{\prime}}\left(S_{\vec{q}_{1}}\left(\vec{y}, t ; \vec{x}_{i}, 0\right), S_{\vec{q}_{2}}\left(\vec{y}, t ; \vec{x}_{j}, 0\right), S_{\vec{q}_{3}}\left(\vec{y}, t ; \vec{x}_{k}, 0\right)\right)
\end{aligned}
$$


By using Eq. (2.7), this new correlation function can be divided two parts as shown in Eq. (2.9). The first part is the summation of the normal correlation function as shown in Eq. (2.2) (using the smeared propagator). The error of these terms will be smaller than Eq. (2.2) with factor $1 / \sqrt{N}$, since we include $N$ source locations. We refer to these as the signal terms in the following discussion. On the other hand, the second part is related to the noise function, and will go to zero when averaged across a large number of noise sources. These noise terms are a source of statistical error for the correlation function. Clearly, to get a better signal, we should make the signal terms stronger and suppress the noise terms, and later we will show how to choose the $N$ source locations toward this aim.

Furthermore, by judiciously choosing the set of three-momenta used to calculate each quark propagator, we can maximise the accessible proton momenta values. Here, we calculate quark propagators with four different values of the three-momenta at the source, $q_{1}=(0,0,0), q_{2}=$ $(0,0,1), q_{3}=(0,1,1)$, and $q_{4}=(1,1,1)$, enabling us to generate 20 different total proton momenta from $(0,0,0)$ to $(3,3,3)$.

\section{Results and Discussions}

\subsection{The error from the noise terms}

The primary source of error in the correlation function $G_{N}$ is from the noise terms. Thus, it is useful to find ways to minimize these terms. In Fig. 1, we show the relative errors of $G_{N}$ for different choices of source locations. The relative errors of correlation function calculated from a single source location (open squares) are larger than those from two source locations as $(0,0,0)$

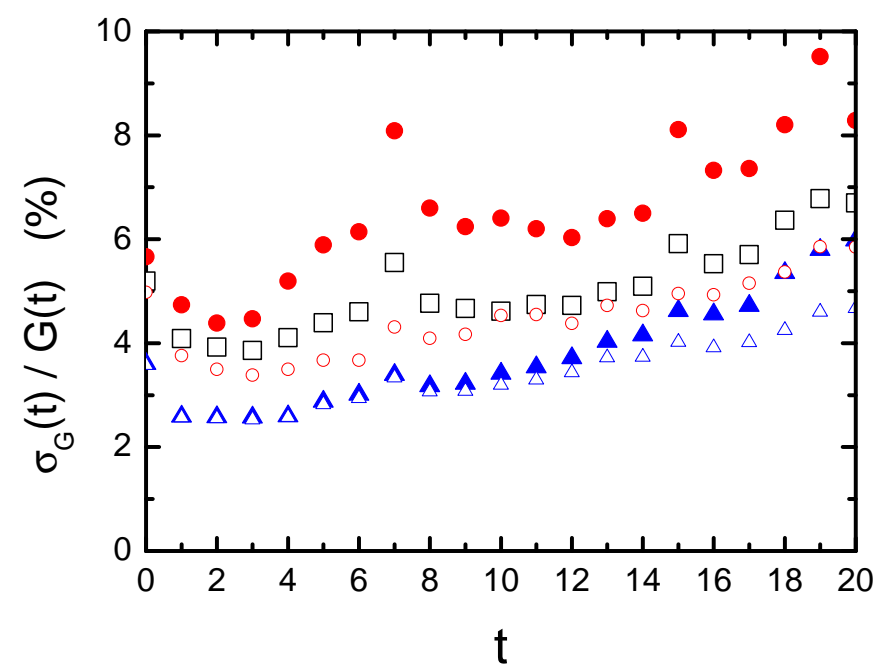

Figure 1: The relative error in the proton correlation function on a $24^{3} \times 48$ lattice. The open squares use a single source location $\vec{x}=(0,0,0)$, while the circles and triangles are calculated using two source points, located at $\vec{x}=(0,0,0),(2,2,2)$ and $\vec{x}=(0,0,0),(12,12,12)$, respectively. The open points represent the signal terms in Eq.(2.9), while the solid points includes both signal and noise terms. 


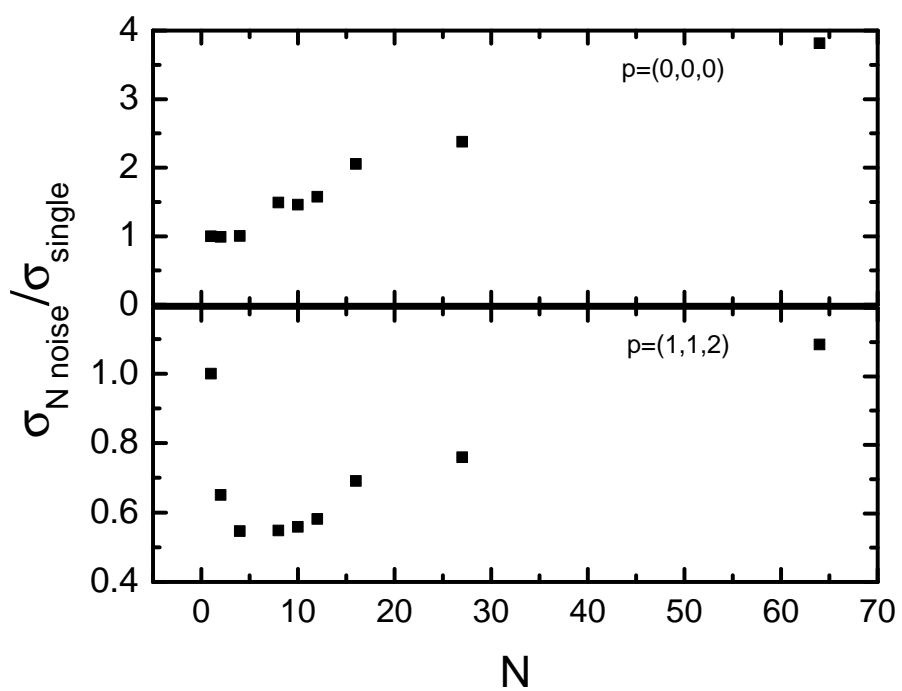

Figure 2: The error in the effective energy of the proton with total momentum $\vec{p} L / 2 \pi=(0,0,0)$ (top) and $(1,1,2)$ (bottom). $N$ is the number of source locations. Error values are normalized to the error of a point source. For each value of $N$, the source locations are chosen to maximize their separation.

and $(2,2,2)$ without noise terms (open circles). However, after including the noise terms (solid circles), the errors increase quickly and grow larger than those of a point source. This enhancement of errors is purely due to the contributions from the noise terms. When we change the two source locations to be further apart at $(0,0,0)$ and $(12,12,12)$ and do the same calculation (triangles), it is clear that the errors both with and without noise terms become similar, and are much smaller than in the previous case (circles). From this comparison, we observe that the distance between source locations will play an important role in minimizing noise terms. This is expected, as the size of the noise terms will be suppressed by the distance between the source points. If the source locations are sufficiently spaced the contribution from noise terms becomes negligible.

To keep the source points appropriately spaced, the number of source locations, $N$, should not be very large. As shown in Eq. (2.9), the number of noise terms is $N^{3}-N$, while the number of signal terms is $N$. This suggests that in each case there exists an optimal choice for $N$. In Fig. 2, the error in the proton effective energy as a function of the number of source points is shown, at zero momentum (top) and at $\vec{P}=(1,1,2)$ (bottom). Maximally separated source locations are chosen for each value of $N$. In the lower plot, at $\vec{P}=(1,1,2)$, we see that as $N$ increases the error drops rapidly and then increases quickly. At this momentum value, for a lattice volume of $24^{3} \times 48$, the best choice is around $N=4-8$.

In summary, the choice of source locations will directly affect the quality of the signal. It is important that the source points are chosen to be sufficiently spaced in order to suppress the contribution from the noise terms.

3.2 Results for $L=24^{3} \times 48$

In Figs. 3 and 4, results are calculated on a lattice volume of $L=24^{3} \times 48$. The black square, 


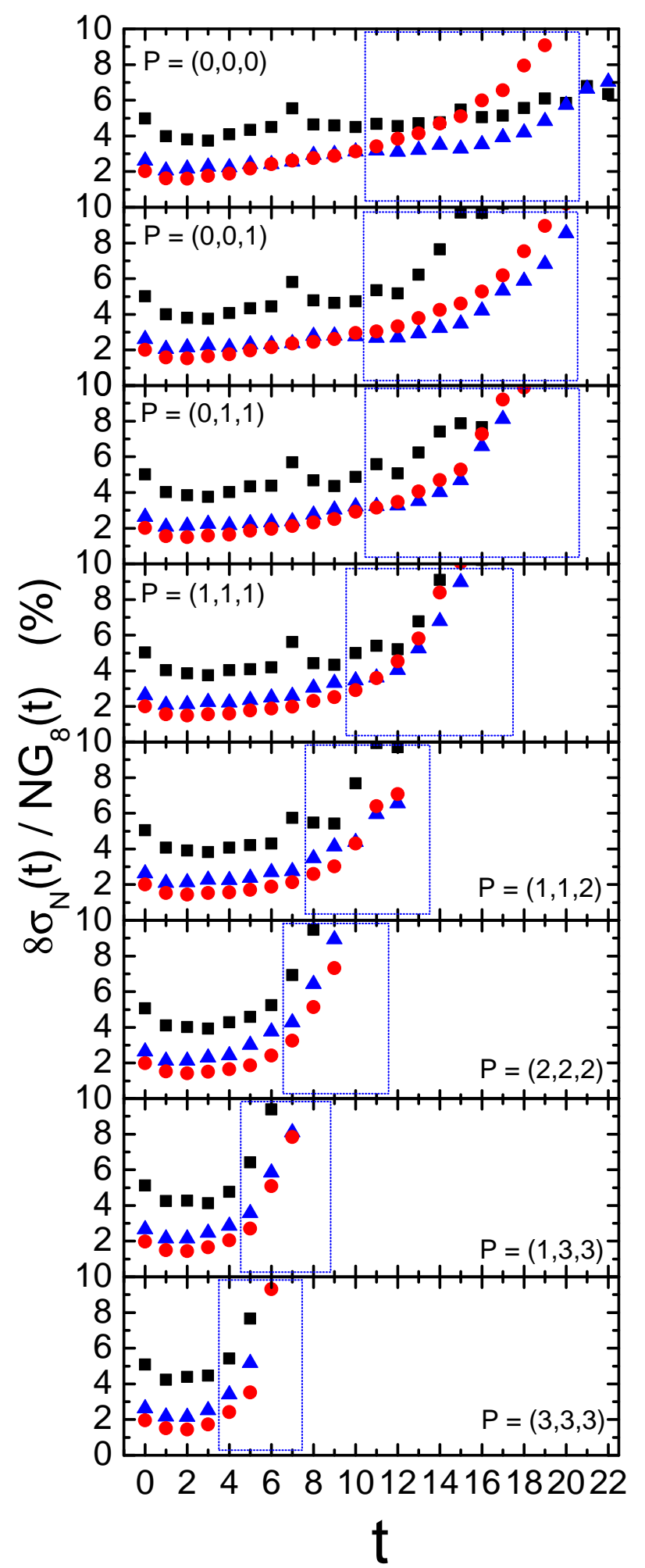

Figure 3: The relative error of the proton correlation function with $N$ source points within a $24^{3} \times 48$ lattice volume. The black squares, blue triangles and red circles represent choices of $N=1,4$ and 8 , respectively. The errors are relative to the central value of the $N=8$ correlation function $G_{8}$. The factor of 8 on the $y$-axis compensates for the use of 8 sources in $G_{8}(t)$. The dashed blue boxes show the selected window for fitting the effective energy. 


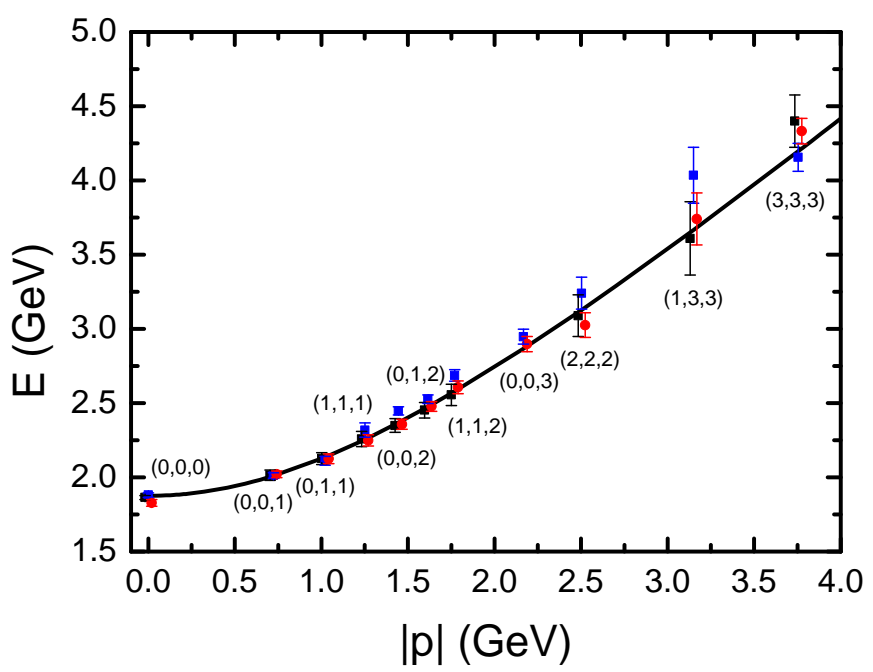

Figure 4: Proton energies for different lattice momenta vs the momentum. The black solid line is calculated from the continuum dispersion. The set of points include those from Fig. 3.

blue triangle and red circle points are calculated with a single source point, 4 sources, and 8sources, respectively. To separate the sources as much as possible, the locations of 4-sources are chosen as the four vertices of a tetrahedron with edge length $12 \sqrt{2}$, and the locations of the 8 sources are the eight vertices of box with edge length 12 .

In Fig. 3, the relative error of the correlation function with different sources is shown. To compare the absolute errors in each case, the relative error is constructed using the central value of the correlation function for 8 -sources, since overall that provides the best signal here. We note that, as shown in Eq. (2.9), the correlation function for $N$-sources is larger than that of a single source by a factor $N$, if the noise terms are negligible, i.e., $G_{N} \sim N G_{1}$. Hence, we divide $G_{8}$, the correlation function for 8-sources, by a factor of 8 , whilst the error $\sigma_{N}$ is divided by a factor of $N$. In this way, the value of $\left(8 \sigma_{N}\right) /\left(N G_{8}\right)$ does not only indicate the relative error of the correlation function, but can also be used to directly compared the absolute value of the error with different source choices. Firstly, we note that at the small $t$, the error of with eight source points is much smaller than that of a single source, by at least a factor of 2 .

However, as Euclidean time increases, the relative error of all three cases increase, with the multi-source error increasing faster. This is understood by noting that the noise terms in the correlation function which originating from the different source locations will grow larger with time evolution, as the extent of the wave function of the quark expands.

Thus, our method provides a much more precise signal at early times. In Fig. 3, the selected fitting ranges for each momentum are shown by the blue box. In these fitting ranges, with the exception of the rest frame, using four and eight source locations gives a better signal than a single source point for each nontrivial momentum value. Through this comparison, we find that the using an appropriate dilute $\mathbb{Z}_{3}$ noise source can provide a significant benefit in obtaining the effective energy of proton in a boosted frame, relative to a standard point source. 
Fig. 4 shows the energy dispersion relation for the proton. In addition to the eight different proton momenta shown in Fig. 3, we also add another three momenta values, $\vec{P}=(0,0,2),(0,1,2)$, and $(0,0,3)$. Unfortunately, for the $\vec{P}=(0,0,3)$ case, we are not able to find an appropriate fit window in the case of a single point source. The black solid line is calculated from the continuum dispersion relation, and is consistent with the lattice results. We can see that the results using eight source points (red circles) provide a very clean energy dispersion all the way up to a proton momentum value of $\vec{P}=(3,3,3)$.

\section{Summary}

We have introduced a novel stochastic quark source using $\mathbb{Z}_{3}$ noise placed on a dilute grid of lattice points, incorporating iterative momentum-smearing. The corresponding correlation function is shown to include two parts, one arising from the signal terms and the other from the noise terms. The signal terms are the summation of various single point source correlation functions, which can provide a more accurate signal than a single point source. Through numerical calculation, we find that there are two ways to reduce the statistical uncertainties that arise from the noise terms. The first is to maximize the separation of the selected source points. The second is to choose an optimal number of source points, since the number of noise terms increases much faster with $N$ than the number of signal terms.

We performed calculations at various momenta on a lattice volume of $24^{3} \times 48$ with sources at 3 choices of $N$ : 4-points, 8-points and a single point source. Momentum-smearing [4] is applied at the source and the sink to reduce the statistical error. We find that for boosted systems, using multiple source locations provides a better signal than that using a single source point. Using a dilute noise source, we can obtain an acceptable signal for values of the total proton momentum up to $\vec{P}=(3,3,3)$, in contrast to a standard source which is unable to provide a usable correlation function at these large momenta. The use of the dilute noise source technique introduced here will extend our ability to perform lattice calculations in boosted frames with high momentum.

\section{References}

[1] S. M. Ryan, Lect. Notes Phys. 889, 35 (2015).

[2] A. L. Kiratidis, W. Kamleh, D. B. Leinweber and B. J. Owen, Phys. Rev. D 91, 094509 (2015)

[3] F. M. Stokes, W. Kamleh, D. B. Leinweber, M. S. Mahbub, B. J. Menadue and B. J. Owen, Phys. Rev. D 92, no. 11, 114506 (2015)

[4] G. S. Bali, B. Lang, B. U. Musch and A. Schäfer, Phys. Rev. D 93, no. 9, 094515 (2016)

[5] R. Babich, R. C. Brower, M. A. Clark, G. T. Fleming, J. C. Osborn, C. Rebbi and D. Schaich, Phys. Rev. D 85, 054510 (2012)

[6] G. S. Bali et al. [QCDSF Collaboration], Phys. Rev. Lett. 108, 222001 (2012)

[7] M. Engelhardt, Phys. Rev. D 86, 114510 (2012)

[8] A. Abdel-Rehim, C. Alexandrou, M. Constantinou, V. Drach, K. Hadjiyiannakou, K. Jansen, G. Koutsou and A. Vaquero, Phys. Rev. D 89, no. 3, 034501 (2014) 
[9] C. McNeile et al. [UKQCD Collaboration], Phys. Rev. D 73, 074506 (2006) doi:10.1103/PhysRevD.73.074506 [hep-lat/0603007].

[10] P. A. Boyle, A. Juttner, C. Kelly and R. D. Kenway, JHEP 0808, 086 (2008)

[11] A. Li et al. [xQCD Collaboration], Phys. Rev. D 82, 114501 (2010) doi:10.1103/PhysRevD.82.114501 [arXiv:1005.5424 [hep-lat]]. 